\title{
nEXO - Neutrinoless double beta decay experiment
}

\section{Brian Mong* for the nEXO Collaboration}

SLAC Accelerator Laboratory

E-mail: bungeslac.stanford.edu

The nEXO collaboration is currently designing a 5-ton liquid-xenon time projection chamber to search for neutrinoless double beta decay. An observation of this decay would show that neutrinos are Majorana particles and that lepton number is not always conserved. Here we discuss some of the initial design choices for the nEXO detector, which will follow the currently operating $\sim 175 \mathrm{~kg}$ EXO-200 experiment. Initial simulations will be presented that show that the nEXO detector will have very low backgrounds and that the multi-parameter approach tested in EXO200 will be even more powerful in the larger nEXO detector.

XIII International Conference on Heavy Quarks and Leptons

22-27 May, 2016

Blacksburg, Virginia, USA

${ }^{*}$ Speaker. 


\section{Introduction}

Several experiments are searching for a rare decay that can only occur if neutrinos are Majorana in nature, known as neutrinoless double beta decay $(0 v \beta \beta)$. In the standard two neutrino double beta decay $(2 v \beta \beta)$, two neutrons in the nucleus decay to protons emitting two electrons and two anti-neutrinos. About a dozen isotopes have measured $2 v \beta \beta$ half-lives, the most recent addition being ${ }^{136} \mathrm{Xe}$ by EXO-200 [1]. If neutrinos are Majorana then it is also possible for decay to occur without emitting any neutrinos, violating lepton number conservation in the process; this is the $0 v \beta \beta$ mode. Detecting $0 v \beta \beta$ requires a large isotopic mass in a detector with very low backgrounds and sufficient energy resolution to discriminate from the $2 v \beta \beta$ mode.

While neutrino oscillations have determined neutrinos are massive, the absolute masses are not known. This is because (in vacuum) neutrino oscillations are only sensitive to the magnitude of the neutrino mass differences. If the $0 v \beta \beta$ half-life can be determined, it contains information on the absolute neutrino mass via

$$
\left(T_{1 / 2}^{0 v}\right)^{-1}=G^{0 v}\left|M^{0 v}\right|^{2}\left|\left\langle m_{\beta \beta}\right\rangle\right|^{2}
$$

where $G^{0 v}$ is a phase space factor and $M^{0 v}$ is the nuclear matrix element. The $m_{\beta \beta}$ is related to the individual neutrino masses $\left(m_{i}\right)$ through the PMNS neutrino mixing matrix $(U)$ by

$$
\left\langle m_{\beta \beta}\right\rangle=\left|\sum_{i=1}^{3} U_{\alpha i}^{2} m_{i}\right| .
$$

Neutrino masses are already problematic for the standard model of particle physics, and neutrinos are natural candidates to be Majorana particles thanks to their chargeless nature. If neutrinos are indeed Majorana in nature, they may be the key to understanding how the asymmetry of matter to antimatter in the universe came about. Because of this profound question, The 2015 Long Range Plan released by the Nuclear Science Advisory Committee has recommended that the US lead a ton-scale $0 v \beta \beta$ experiment in a timely manner [2].

\section{2. nEXO Sensitivity}

The $\mathrm{nEXO}$ collaboration is designing a $\sim 5000 \mathrm{~kg}$ liquid xenon (LXe) time projection chamber (TPC) to search for the $0 v \beta \beta$ decay mode in ${ }^{136} \mathrm{Xe}$. The goal for $\mathrm{nEXO}$ is to completely cover the inverted mass hierarchy and to push into the normal hierarchy as shown in Fig. 1. Also shown is the sensitivity of the predecessor experiment, EXO-200 (at $175 \mathrm{~kg}$ ), in the most recent analysis $[3]$ as well as its projected final sensitivity. The nEXO experiment is designed to be flexible, with multiple options available depending on what is found in the initial 5 year run period. If a $0 v \beta \beta$ like signal is observed, the enriched LXe can be replaced with LXe depleted in ${ }^{136} \mathrm{Xe}$ to confirm the discovery, and the enriched Xe can be used in a gas detector to study the electron correlations in $0 v \beta \beta$ to understand the underlying mechanism ( [4]). On the other hand, if $0 v \beta \beta$ is not observed, the enriched LXe can be reused in a larger detector or the detector itself can be upgraded to increase sensitivity. Barium tagging is one such upgrade that the $\mathrm{nEXO}$ collaboration is actively researching to increase sensitivity. With tagging, the daughter of $0 v \beta \beta$ decays $\left({ }^{136} \mathrm{Ba}\right)$ are identified by atomic 


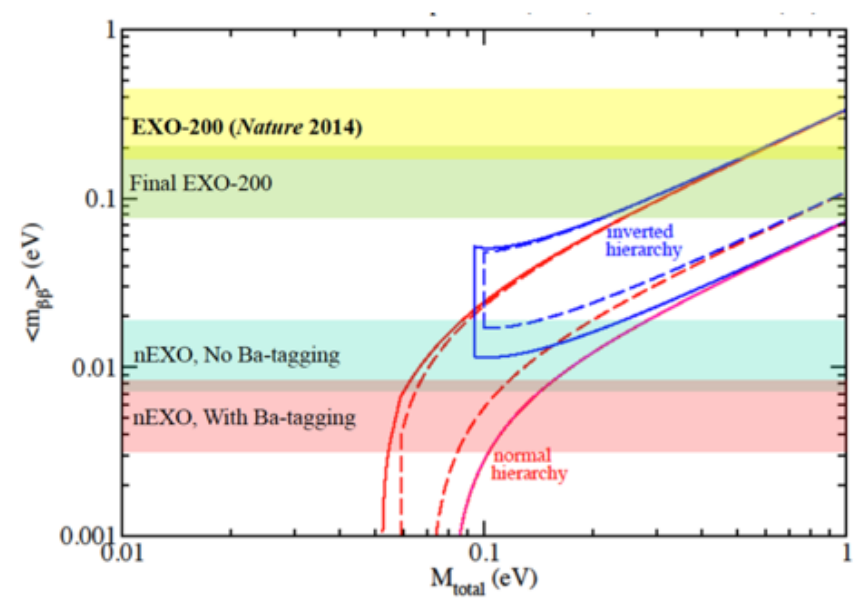

Figure 1: Sensitivity for EXO-200 and nEXO in terms of the Majorana neutrino mass assuming a range of nuclear matrix elements.

spectroscopy, thereby confirming the event from a double beta decay (see [5], [6], and [7] for more information). The expected sensitivity of nEXO with barium tagging is also shown in Fig. 1.

The predecessor experiment to $\mathrm{nEXO}, \mathrm{EXO}-200$, has demonstrated the power of a large monolithic TPC detector using a multi-parameter search for $0 v \beta \beta$. EXO-200 has made the first $2 v \beta \beta$ measurement of ${ }^{136} \mathrm{Xe}$ [1], the most precise $2 v \beta \beta$ half-life measurement of any isotope [8], and set a stringent limit on $0 v \beta \beta$ in excess of $1.1 \times 10^{25}$ years [3]. This power comes from using the combination of ionization density, energy resolution, event multiplicity, and event location to discriminate $0 v \beta \beta$ from backgrounds. EXO-200 has also been a success by validating the background model that was used during construction, meeting the design background goals [9].

\section{3. nEXO R\&D}

The nEXO collaboration has begun working on the initial detector design which includes a number of technological improvements over EXO-200. There are also R\&D projects looking at the challenges of scaling up the detector size, such as higher voltage and purity requirements as well as novel calibration techniques to reach deep inside the volume. Fig. 2 shows the EXO-200 detector next to a preliminary design of the nEXO detector. Prominent changes include the central cathode of EXO-200, which has been moved in nEXO to create one large drift region to maximize the volume of low background xenon in the center of the detector, and the photo-detectors have been moved to the barrel for greater coverage and to allow for a new charge collection system that uses opaque pads rather than crossed wires at the anode. A few of the new technologies that are being investigated by the collaboration include:

- VUV-SiPM photodetectors for improved energy resolution and lower radioactivity [10],

- Charge collection pads for finer position resolution and lower thresholds,

- In LXe digitization electronics for improved energy resolution and lower energy thresholds, 


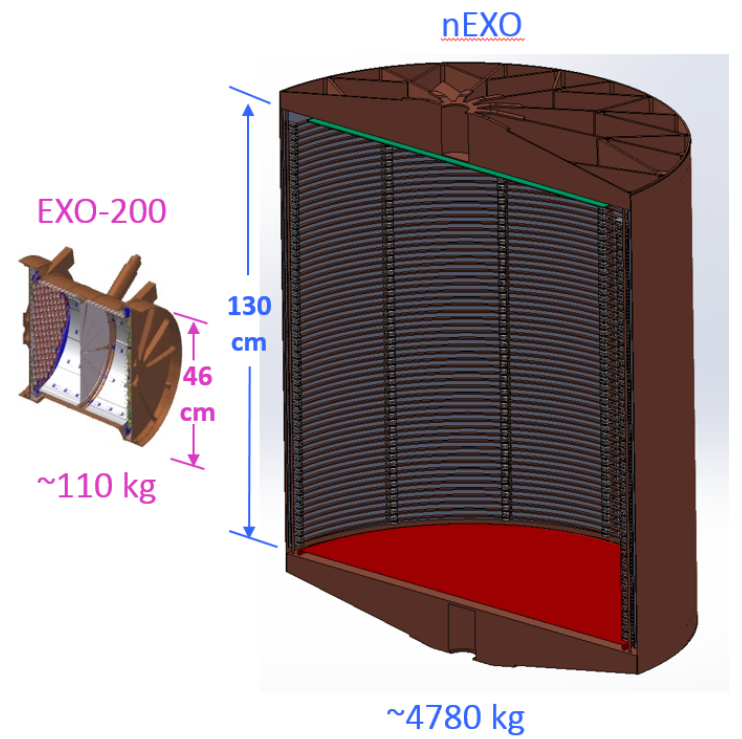

Figure 2: EXO-200 and the preliminary nEXO design shown side by side for comparison. EXO-200 has two drift volumes that share a common central cathode. The nEXO detector has a cathode (green) at one end and charge collecting pads for the anode (red) at the other end. Photodetectors (grey) are shown along the barrel. EXO-200 uses teflon as a reflective surface to reflect scintillation and APDs at the anodes measure scintillation.

- Improve radio-assay sensitivities and begin measuring potential construction materials,

- No plastics in the detector for lower outgassing and higher purity.

With these improvements, nEXO is expected to have $1 \%$ (or better) energy resolution at the $0 \mathrm{v} \beta \beta$ Q-value $(2457 \mathrm{keV})$.

Monte-Carlo simulations have also begun to test a number of design questions. These simulations have helped develop radio-purity requirements for construction as well as have been useful in determining performance requirements to meet the sensitivity goals of nEXO. In Fig. 3 simulated energy spectra are shown for successively smaller fiducial volumes in both single site and multi-site type events. These simulations show the benefit of a large monolithic detector that can use the outer LXe to measure the backgrounds while shielding the central volume. In the analysis, backgrounds are fit with the position information to optimally use the self shielding nature of the detector. The advantage of a large monolithic detector can also be seen in the estimated background index for nEXO, shown in Fig. 4. Since radioactive backgrounds originate from the detector materials, the inner volumes of LXe that are being shielded by the outer xenon see very low backgrounds.

\section{Summary}

The nEXO collaboration is working on the design of a 5 ton LXe TPC to search for $0 v \beta \beta$ beyond the inverted neutrino hierarchy. With a larger monolithic detector, the multi-parameter 


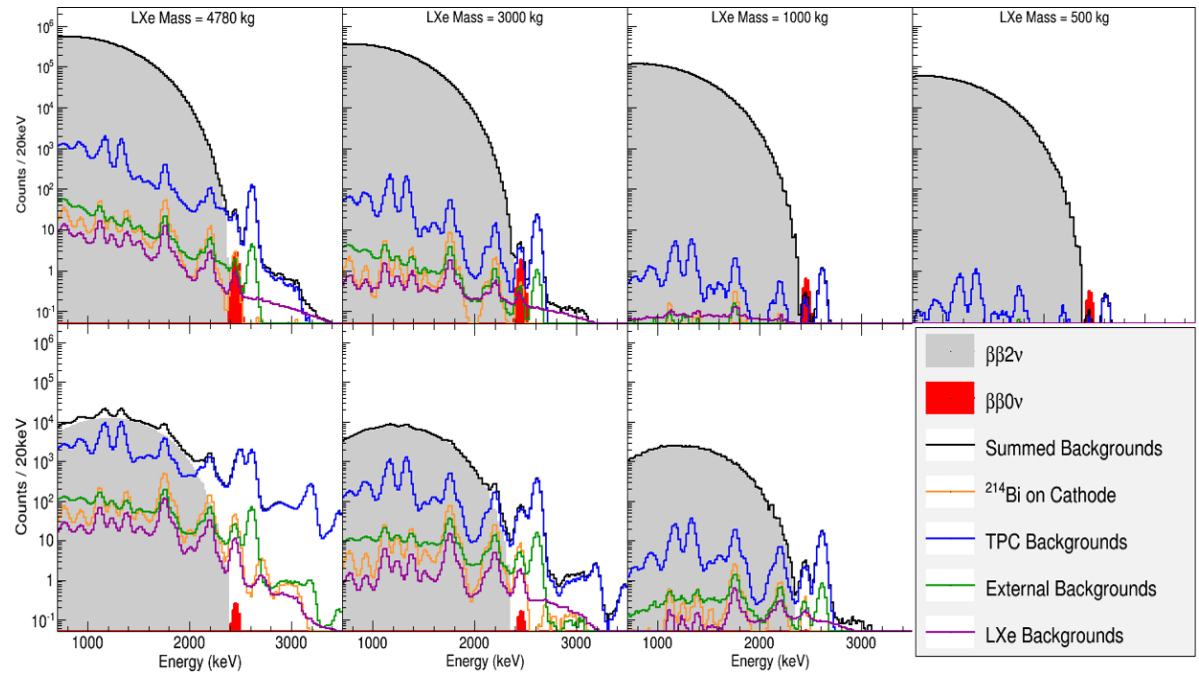

Figure 3: Simulated nEXO spectra for single site (top row) and multi-site (bottom row) for different fiducial volumes. Shown with $T_{1 / 2}^{0 v \beta \beta}=6.6 \times 10^{27} \mathrm{yr}$.

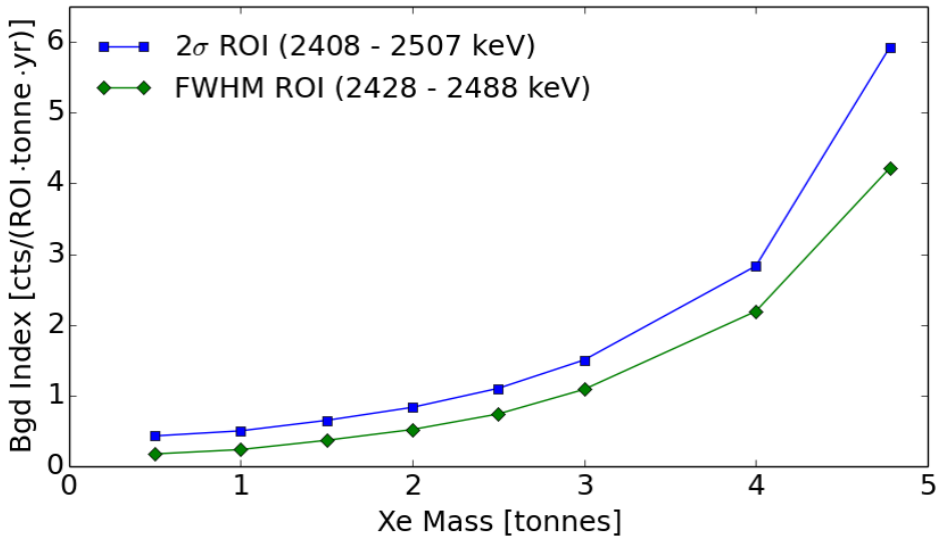

Figure 4: Estimate of the background index of $\mathrm{nEXO}$ as a function of fiducial volume in the $2 \sigma$ ROI.

analysis tested in EXO-200 will be even more powerful. Presently a large number of R\&D projects are underway to test new technologies for the nEXO detector.

\section{References}

[1] N. Ackerman, et al., Observation of two-neutrino double-beta decay in ${ }^{136}$ Xe with the exo-200 detector, Phys. Rev. Lett. 107 (2011) 212501.

[2] The 2015 Nuclear Science Advisory Committee, The 2015 long range plan for nuclear science. URL http://science.energy.gov/ /media/np/nsac/pdf/2015LRP/2015_ LRPNS_091815.pdf 
[3] J. B. Albert, et al., Search for majorana neutrinos with the first two years of exo-200 data, Nature 510 (2014) $229-234$.

[4] A. Ali, A. V. Borisov, D. V. Zhuridov, Probing new physics in the neutrinoless double beta decay using electron angular correlation, Phys. Rev. D 76 (2007) 093009.

[5] K. Twelker, et al., An apparatus to manipulate and identify individual ba ions from bulk liquid xe, Review of Scientific Instruments 85 (9).

[6] B. Mong, et al., Spectroscopy of ba and $\mathrm{ba}^{+}$deposits in solid xenon for barium tagging in nexo, Phys. Rev. A 91 (2015) 022505.

[7] J. B. Albert, et al., Measurements of the ion fraction and mobility of $\alpha$ - and $\beta$-decay products in liquid xenon using the exo-200 detector, Phys. Rev. C 92 (2015) 045504.

[8] J. B. Albert, et al., Improved measurement of the $2 v \beta \beta$ half-life of ${ }^{136}$ xe with the exo-200 detector, Phys. Rev. C 89 (2014) 015502.

[9] J. B. Albert, et al., Investigation of radioactivity-induced backgrounds in exo-200, Phys. Rev. C 92 (2015) 015503.

[10] I. Ostrovskiy, et al., Characterization of silicon photomultipliers for nexo, IEEE Transactions on Nuclear Science 62 (4) (2015) 1825-1836. 